\title{
Gain of Regularity in Extension Problem on Convex Domains
}

\author{
M. Jasiczak \\ Faculty of Mathematics and Computer Science, Adam Mickiewicz University, Ulica Umultowska 87, 61-614 Poznan, Poland
}

Correspondence should be addressed to M. Jasiczak; mjk@amu.edu.pl

Received 19 March 2015; Accepted 18 June 2015

Academic Editor: Alberto Fiorenza

Copyright (C) 2015 M. Jasiczak. This is an open access article distributed under the Creative Commons Attribution License, which permits unrestricted use, distribution, and reproduction in any medium, provided the original work is properly cited.

We investigate the extension problem from higher codimensional linear subvarieties on convex domains of finite type. We prove that there exists a constant $d$ such that on Bergman spaces $H^{p}(D)$ with $1 \leq p<d$ there appears the so-called "gain regularity." The constant $d$ depends on the minimum of the dimension and the codimension of the subvariety. This means that the space of functions which admit an extension to a function in the Bergman space $H^{p}(D)$ is strictly larger than $H^{p}(D \cap A)$, where $A$ is a subvariety.

\section{Introduction}

Let $D$ be a bounded pseudoconvex domain, $\psi: D \rightarrow \mathbb{R} \cup$ $\{-\infty\}$ a plurisubharmonic function, and $A \subset \mathbb{C}^{n}$ a complex linear hyperplane. In [1], Ohsawa and Takegoshi proved that there is a constant $C$ depending only on the diameter of $D$ such that for any function $f$ holomorphic in $D \cap A$ with

$$
\int_{D \cap A}|f|^{2} e^{-\psi} d V_{D \cap A}<\infty
$$

there is a function $F \in H(D)$ satisfying $\left.F\right|_{D \cap A}=f$ and

$$
\int_{D}|F|^{2} e^{-\psi} d V \leq C \int_{D \cap A}|f|^{2} e^{-\psi} d V_{D \cap A}
$$

The symbol $d V_{D \cap A}$ stands for the measure induced on $D \cap$ $A$ by the volume element of $D \cap A$, that is, the $(2 n-2)$ dimensional Lebesgue measure, $d V$, is the volume measure in $\mathbb{C}^{n}$. This result turned out to be extremely useful in both complex analysis and complex geometry (cf. references in [2] and a recent article by Demailly et al. [3]). Not surprisingly, it stimulated a lot of research. There are at least three natural ways of generalizing the Ohsawa-Takegoshi Theorem. The first one is to study the extension problem on different function spaces, for instance other $L^{p}$ spaces, with the prominent case of bounded extensions of bounded holomorphic functions (for strictly pseudoconvex domains, this problem was solved by Henkin [4] and Amar [5], for convex finite type domains and linear affine subvarieties by Diederich and Mazzilli in [6], and the case of varieties which are not necessarily linear was studied by Alexandre in [7]). The second one is to investigate dependence of the problem on the geometry of $D$ or $b D$. Lastly, it is also natural to ask when the extension can be realized by a linear operator.

Cumenge in [8] considered the extension problem in case of strictly pseudoconvex domains and subvarieties $A$ of codimension $k \geq 1$ which are nonsingular and cut $b D$ transversally. She proved that in this case any holomorphic function in $L^{p}\left((-r)^{s} d V_{D \cap A}\right), s \geq k, 1 \leq p<\infty$, admits an extension to a holomorphic function which belongs to $L^{p}\left((-r)^{s-k} d V\right)$. The symbol $r$ stands for a function which defines the domain $D$. Such a function is uniformly comparable with $\operatorname{dist}(\cdot, b D)$, when $b D$ is smooth.

In order to motivate our study, let us restrict our attention to the $L^{2}$ case. Comparing with the Ohsawa-Takegoshi Theorem for $\psi=0$, the result of Cumenge says that there is a strictly larger class of functions than $L^{2}(D \cap A) \cap H(D)$ which admit an extension to a holomorphic function in $L^{2}(D)$. There appears the so-called "gain of regularity." Observe also that each complex codimension counts as one in the exponent of the weight $(-r)^{s}$. We say that the problem is isotropic in the case of strictly pseudoconvex domains. Such phenomena have been central in the whole $\bar{\partial}$-problem theory and PDE's in general (a natural example here is subelliptic estimates [9-12] on finite type domains). 
It came therefore as a surprise when Diederich and Mazzilli proved in [2] that there are elementary pseudoconvex domains and subvarieties with no "regularity gain." Specifically, they proved that for arbitrary integers $p \geq 1,2 \leq$ $q \leq \infty$ and for any $\varepsilon>0$ there is a bounded pseudoconvex domain $D \subset \mathbb{C}^{2 p+1}$ with smooth polynomial boundary and a function $f \in H^{q}\left(D \cap A_{p}\right)$, where

$$
A_{p}:=\left\{z \in \mathbb{C}^{2 p+1}: z_{1}=\cdots=z_{p}=0\right\},
$$

such that $f$ does not have a holomorphic extension in $H^{2 q(p+2) /(q p+4)+\varepsilon}(D)$ (resp. $H^{2(p+2) / p+\varepsilon}(D)$ for $q=\infty$ ). This result implies in particular that for each $\varepsilon>0$ there is a positive integer $p$ and a bounded pseudoconvex domain $D \subset \mathbb{C}^{2 p+1}$ with smooth polynomial boundary, such that $A_{p}$ intersects $b D$ transversally at all points $b D \cap A_{p}$ and such that there is a holomorphic function $f \in H^{\infty}\left(D \cap A_{p}\right)$, not admitting an extension in $H^{2+\varepsilon}(D)$. It seems important to notice that the minimum of the dimension and the codimension of the subvariety $A_{p}$ is maximally possible.

In this paper, we consider bounded convex domains of finite type, so in particular domains considered by Diederich and Mazzilli in [2], and complex linear subvarieties of higher codimension. We prove a result which goes in the opposite direction comparing with the results of Diederich and Mazzilli. Namely, on such domains and subvarieties there always is the "gain of regularity" in the extension problem. We will make clear this statement below.

In order to present the result, we need however some definitions. For $1 \leq m \leq n-1$ consider linear affine mappings

$$
l_{i}(z)=a_{i 1} z_{1}+\cdots+a_{i n} z_{n}+b_{i}, \quad i=1, \ldots, m,
$$

where $a_{i j}, b_{i} \in \mathbb{C}, i=1, \ldots, m, j=1, \ldots, n$. Let $A=$ $A\left(l_{1}, \ldots, l_{m}\right)$ be the joint zero set of $l_{1}, \ldots, l_{m}$. Assume that $D \cap A \neq \emptyset$ and $\operatorname{dim} A=n-m$. For a positive Borel measure $\omega$ supported on $D \cap A$, we write $H^{p}(D \cap A, \omega)$ to denote the space of all functions $f$ holomorphic in $D \cap A$ such that

$$
\int_{D \cap A}|f|^{p} d \omega<\infty
$$

We can now present the main result.

Theorem 1. Assume that $D \subset \mathbb{C}^{n}$ is a bounded convex domain of finite type defined by a smooth function $r$ such that $d r \neq 0$ on $b D$. If $p>1$ and

$$
1+\frac{1}{p-1}>\min \{n-m, m\}>1
$$

then there exists a positive Borel measure $\omega$ supported on $D \cap A$ such that

$$
H^{p}(D \cap A) \subsetneq H^{p}(D \cap A, \omega)
$$

and an operator

$$
\begin{gathered}
E_{D \cap A}: H^{p}(D \cap A, \omega) \longrightarrow H^{p}(D) \\
\text { satisfying }\left.E_{D \cap A} f\right|_{D \cap A}=f \text { for any } f \in H^{p}(D \cap A, \omega) .
\end{gathered}
$$

In other words, if for $p>1$ it holds that $1+1 /(p-1)>$ $\min \{n-m, m\}>1$, then the class of functions which admit an extension in $H^{p}(D)$ is strictly larger than $H^{p}(D \cap A)$. Also for any dimension of $A$ there is also a space $H^{p}(D)$ with $p>1$ where the "gain of regularity" appears.

We also establish the following result.

Theorem 2. Assume that $D \subset \mathbb{C}^{n}$ is a bounded convex domain of finite type defined by a smooth function $r$ such that $d r \neq 0$ on $b D$. There exists a positive Borel measure $\omega$ supported on $D \cap A$ such that

$$
H^{1}(D \cap A) \varsubsetneqq H^{1}(D \cap A, \omega)
$$

and an operator

$$
E_{D \cap A}: H^{1}(D \cap A, \omega) \longrightarrow H^{1}(D)
$$

satisfying $\left.E_{D \cap A} f\right|_{D \cap A}=f$ for any $f \in H^{1}(D \cap A, \omega)$.

Thus, the space of functions which admit an extension in $H^{1}(D)$ is always larger than $H^{1}(D \cap A)$.

It is natural to ask what happens when condition (6) is not satisfied. We do not have any answer yet. What is clear however is that the estimates which we provide do not work anymore. Another natural and closely related question is whether the operator $E_{D \cap A}$ maps $H^{p}(D \cap A)$ into $H^{p}(D)$ for any $p$ between 1 and $\infty$. In particular, whether $E_{D \cap A}$ maps $H^{2}(D \cap A)$ into $H^{2}(D)$ without any gain. We are only able to prove the following fact.

Theorem 3. Assume that $\min \{m, n-m\} \leq 2$, and then

$$
E_{D \cap A}: H^{p}(D \cap A) \longrightarrow H^{p}(D)
$$

for any $1 \leq p \leq 2$.

In view of Ohsawa-Takegoshi Theorem, it suggests that our method of extending holomorphic functions is not optimal.

Convex finite (d'Angelo 1-) type domains are important class of domains where geometric aspects of function theory are studied. The finite type conditions were discovered in connection with the $\bar{\partial}$-Neumann problem (see the fundamental works of Kohn $[9,10]$ and Catlin $[11,12]$, and see also [13] for more information on the type condition). By the results in $[14,15]$, the assumption that a convex domain is of finite d'Angelo 1-type is equivalent to the assumption that there exists a constant $M$ such that all complex lines have order of contact at most $M$ with $b D$ (cf. also [16] for an important generalization of this property for the multitype).

There are a few cornerstones in the study of function theory on convex domains. The first step was made by Bruna et al. [17] and McNeal $[15,18]$ who introduced the correct notion of pseudoballs and pseudometric on such domains. This was used to describe boundary behaviour of the Bergman kernel by McNeal [18] and the Szegö kernel by McNeal and Stein [19]. The fundamental step was made by Bruna et al. in [20]. The authors showed op. cit. how to estimate kernel functions in terms of boundary distances. 
Another breakthrough came when Diederich and Fornæss [21] constructed support functions for convex finite type domains. This made it possible to answer many analytic questions such as the quantitative behaviour of the $\bar{\partial}$-equation on $L^{p}$ spaces [22-24] and Hölder spaces $[25,26]$. This made it also possible to study extension problems by means of integral operators, for instance by means of operators constructed by Berndtsson and Andersson [27] and Berndtsson [28]. As we have already written, this was started by Diederich and Mazzilli in [6]. We continue this task in this paper. We mention also that other aspects of function theory on convex finite type domains such as duality problems were also studied [29]. We remark that recently Nikolov et al. [30] found a mistake in $[15,18]$. This however has no influence on our work since crucial estimates, in particular formula (49) below, remain valid.

\section{Proof}

Let $D=\{r<0\}$ be a bounded convex domain with $C^{\infty}$ boundary of finite type $M$. We may assume that $r$ has been chosen to be convex on $\mathbb{C}^{n}$ and smooth in $\mathbb{C}^{n} \backslash\{0\}$. We assume that the domain $D$ is of type $M$. As was stated in the Introduction section, this means that the maximal order of contact of $b D$ with complex lines is equal at most $M$.

In order to prove Theorems 1 and 2, we use an extension operator $E_{D \cap A}^{N}$ constructed by Berndtsson in [28]the construction relies on previous results by Berndtsson and Andersson in [27]. For the machinery developed by Berndtsson and Andersson to work, we need appropriate holomorphic support functions. Such functions, depending smoothly on $\zeta \in D$ and of optimal contact behaviour, were constructed by Diederich and Fornæss in [21]. The paper [25] contains crucial estimates, which we will use in the proofs. Since such estimates were used by many authors before, most notably by Diederich et al. in [25] and Fischer in [22, 26], we are rather brief in this aspect of the proof. For the same reason, we do not include separate background on geometry of convex finite type domains. Such information can naturally be found in papers by McNeal $[15,18]$. It was also given in many papers on convex finite type domains; we refer the reader for instance to [20] or [25].

The extension operator $E_{D \cap A}^{N}$ is an integral operator of the form

$$
E_{D \cap A}^{N} f(z)=\int_{D \cap A} f(\zeta) E_{D \cap A}^{\mathrm{N}}(\zeta, z) d V_{D \cap A}(\zeta)
$$

defined by a kernel function $E_{D \cap A}^{N}(\cdot, \cdot)$.

The proof of boundedness of $E_{D \cap A}^{N}$ on $L^{p}$ spaces in Theorem 1 is based on the following modification of Schur's test.

Proposition 4. Let $\mu, \nu$ be positive Borel measures on $X$ and let $W$ be a positive weight function. If there exist nonnegative functions $h_{1}, h_{2}$ such that

$$
\begin{array}{r}
\int_{X} K(x, y) h_{1}(y)^{q} W^{-q / p}(y) d \mu(y) \leq C_{1} h_{2}(x)^{q}, \\
\int_{X} K(x, y) h_{2}(x)^{p} d \nu(x) \leq C_{2} h_{1}(y)^{p},
\end{array}
$$

then the operator

$$
T f(x)=\int_{X} f(y) K(x, y) d \mu(y)
$$

is a bounded operator between $L^{p}(X, W d \mu)$ and $L^{p}(X, d \nu)$.

The proof of Proposition 4 is an easy modification of the standard case, which can be found for instance in [31] p. 52. Therefore, we omit it.

Sufficiently close to the boundary of the domain $D$, and only this is the case of interest, the extension operator $E_{D \cap A}$ takes the form

$$
\begin{aligned}
& E_{D \cap A}^{N} f(z):=-\frac{(n-1) !}{m !(n-m) !} \int_{D \cap A\left(l_{1}, \ldots, l_{m}\right)} f(\zeta) \\
& \left.\quad \cdot\left[d V^{\#}\right]\left(\frac{r^{N+n-m}(\zeta)}{(r(\zeta)+S(z, \zeta))^{N+n-m}}\left(\bar{\partial}\left(\frac{1}{r(\zeta)} Q(z, \zeta)\right)\right)^{n-m} \wedge \Omega\left[l_{1}, \ldots, l_{m}\right](\zeta)\right)\right] d V_{D \cap A\left(l_{1}, \ldots, l_{m}\right)}(\zeta),
\end{aligned}
$$

where

$$
\begin{aligned}
& \Omega\left[l_{1}, \ldots, l_{m}\right] \\
& =\frac{\left(\sum_{j=1}^{n} a_{1 j} d \zeta_{j}\right) \wedge \cdots \wedge\left(\sum_{j=1}^{n} a_{m j} d \zeta_{j}\right) \wedge \bar{\partial}_{1} \wedge \cdots \wedge \bar{\partial}_{m}}{\left\|\partial l_{1} \wedge \cdots \wedge \partial l_{m}\right\|^{2}} .
\end{aligned}
$$

Symbol $d V^{\#}$ stands for the $(n, n)$-vector dual to the volume form $d V$ in $\mathbb{C}^{n}$, while $\rfloor$ denotes the contraction between the exterior algebras $\Lambda T$ and $\Lambda T^{*}$ of the tangent and cotangent bundles.
Crucial in the whole construction is the function $S$ which is the support function constructed for convex domains of finite type in [21] by Diederich and Fornæss. Function $S$ is appropriately decomposed, like in Hefer's Lemma, to yield the form

$$
Q(z, \zeta):=\sum_{j=1}^{n} Q_{j}(z, \zeta) d \zeta_{j}
$$

Details of the construction can be found in [25]. 
In order to apply Proposition 4, we need to choose measures $\mu, v$ and functions $W, h_{1}$, and $h_{2}$. Since we are interested in values of the operator $E_{D \cap A}^{N}$ in the space $H^{p}(D)$, we set $d \mu=d V_{D \cap A}$ and $d v=d V$. It remains therefore to find appropriate functions $W, h_{1}$, and $h_{2}$. To accomplish this task, we need to recall some information on convex domains of finite type. Let, as in $[15,18]$,

$$
\begin{aligned}
& \tau(\zeta, v, \varepsilon) \\
& \quad:=\max \{c:|r(\zeta+\lambda v)-r(\zeta)|<\varepsilon \forall \lambda \in \mathbb{C},|\lambda|<c\}
\end{aligned}
$$

be a complex directional boundary distance. For a fixed point $\zeta$ and fixed radius $\varepsilon$, we define the $\varepsilon$-extremal basis $\left(v_{1}^{\zeta, \varepsilon}, \ldots, v_{n}^{\zeta, \varepsilon}\right)$ centered at $\zeta$ as in [15]. Once the basis is chosen, we write $\tau_{j}(\zeta, \varepsilon)$ to denote $\tau\left(\zeta, v_{j}^{\zeta, \varepsilon}, \varepsilon\right)$.

Functions $h_{1}, h_{2}$, and $W$ have to capture the nonisotropic nature of the problem. Following [20], we therefore define the following.

Definition 5. Assume that $D$ is a bounded convex domain of finite type in $\mathbb{C}^{n}, n>1$ defined by a function $r$ which is smooth in $\mathbb{C}^{n} \backslash\{0\}$, convex in $\mathbb{C}^{n}$ and such that $d r \neq 0$ on $b D$. Let $\Omega$ be an $(m, 0)$-covector at $\zeta \in D$. The nonisotropic norm $|\Omega|_{\mathscr{N}}(\zeta)$ of $\Omega$ at $\zeta$ is defined as

$$
\begin{aligned}
& |\Omega|_{\mathcal{N}}(\zeta):=\sup \left\{\left|\Omega\left(v_{1}, \ldots, v_{m}\right)\right|\right. \\
& \left.\cdot \prod_{j=1}^{m} \tau\left(\zeta, v_{j},|r(\zeta)|\right): v_{1}, \ldots, v_{m} \neq 0\right\} .
\end{aligned}
$$

With this definition, we can formulate Lemma 6.

Lemma 6. Assume that $D$ is a bounded convex domain of finite type in $\mathbb{C}^{n}, n>1$ defined by a function $r$ which is smooth in $\mathbb{C}^{n} \backslash\{0\}$, convex in $\mathbb{C}^{n}$ and such that $d r \neq 0$ on $b D$. For the variety $A=A\left(l_{1}, \ldots, l_{m}\right)$, consider the operator $E_{D \cap A}^{N}$. If $N$ is sufficiently large, then there exists a constant $C$ such that

$$
\int_{D}\left|E_{D \cap A}^{N}(\zeta, z)\right| d V(z) \leq C\left|\partial l_{1} \wedge \cdots \wedge \partial l_{m}\right|_{,}^{2}(\zeta)
$$

Proof. We define polydiscs

$$
C P_{\varepsilon}(\zeta):=\left\{z=\zeta+\sum_{j=1}^{n} z_{j, \zeta, \varepsilon} v_{j}:\left|z_{j, \zeta, \varepsilon}\right| \leq C \tau_{j}(\zeta, \varepsilon)\right\}
$$

and for $i \in \mathbb{N}_{0}$ the corresponding polyannuli

$$
P_{\varepsilon}^{i}(\zeta):=C_{1} P_{2^{i} \varepsilon} \backslash \frac{1}{2} P_{2^{i} \varepsilon}(\zeta)
$$

The constant $C_{1}$ is chosen in such a way that $C_{1} P_{\varepsilon / 2}(\zeta)$ J $(1 / 2) P_{\varepsilon}(\zeta)$ for all $\zeta, \varepsilon$ (cf. Proposition 3.1 (ii) in [25]). For simplicity, we assume that $C_{1}=1$.

Then, we have for fixed $\varepsilon_{0}$ that

$$
P_{\varepsilon_{0}}(\zeta) \subset P_{\varepsilon}(\zeta) \cup \bigcup_{i=0}^{C\left[\log _{2}\left(\varepsilon_{0} / \varepsilon\right)\right]} P_{\varepsilon}^{i}(\zeta)
$$

For fixed $\zeta \in D$, we use this cover with $\varepsilon=|r(\zeta)|$ and estimate

$$
\begin{aligned}
& \int_{D}\left|E_{D \cap A}^{N}(\zeta, z)\right| d V(z) \\
& \quad \leq\left\{\int_{D \backslash P_{\varepsilon_{0}}(\zeta)}+\int_{P_{\mid r(\zeta)}(\zeta)}+\sum_{i=0}^{C\left[\log _{2}\left(\varepsilon_{0} /|r(\zeta)|\right)\right]} \int_{P_{\mid r(\zeta)}^{i}}\right\} \\
& \cdot\left|E_{D \cap A}^{N}(\zeta, z)\right| d V(z) .
\end{aligned}
$$

We show how to estimate the integral of a typical term of the kernel function $E_{D \cap A}^{N}(\cdot, \cdot)$ over $P_{|r(\zeta)|}^{i}(\zeta)$. We will keep denoting the typical term by the same symbol $E_{D \cap A}^{N}(\cdot, \cdot)$.

It is a consequence of Lemma 3.2 in [25] that we may assume that

$$
|S(z, \zeta)+r(\zeta)| \gtrsim 2^{i}|r(\zeta)|
$$

in $P_{|r(\zeta)|}^{i}(\zeta)$. Therefore,

$$
\begin{aligned}
& \left|E_{D \cap A}^{N}(\zeta, z)\right| \leq C\left(2^{i}\right)^{-N}\left(2^{i}|r(\zeta)|\right)^{-(n-m)} \\
& \left.\cdot \mid(d V)^{\#}\right]\left(\sum_{j=1}^{n} \bar{\partial} Q_{j}(\zeta, z) \wedge d \zeta_{j}\right)^{n-m} \\
& \quad \wedge \Omega\left[l_{1}, \ldots, l_{m}\right] \mid
\end{aligned}
$$

for $z \in P_{|r(\zeta)|}^{i}(\zeta)$. In order to estimate the right-hand side in (26), we choose the $2^{i}|r(\zeta)|$-extremal basis $\left(v_{1}, \ldots, v_{n}\right)=$ $\left(v_{1}^{\zeta, 2^{i}|r(\zeta)|}, \ldots, v_{n}^{\zeta, 2^{i}|r(\zeta)|}\right)$ at $\zeta$. Let $\left(w_{1}, \ldots, w_{n}\right)$ be the corresponding coordinates of a point $z$ and let $\Phi$ be a unitary transformation such that $z=\Phi(w)+\zeta:=\varphi(w)$; that is, $w=\Phi^{\star}(z-\zeta)$. We use functorial properties of the contraction operation 


$$
\begin{aligned}
& \left.\left((d V)^{\#}\right\rfloor\left(\left(\sum_{j=1}^{n} \bar{\partial} Q_{j} d \zeta_{j}\right)^{n-m} \wedge \Omega\left[l_{1}, \ldots, l_{m}\right]\right)\right)(\zeta, z) \\
& \left.=\left((d V)^{\#}\right\rfloor\left(\left(\sum_{j=1}^{n} \bar{\partial} Q_{j} d \zeta_{j}\right)^{n-m} \wedge \Omega\left[l_{1}, \ldots, l_{m}\right]\right)\right)\left(\varphi \varphi^{-1}(\zeta), \varphi \varphi^{-1}(z)\right) \\
& \left.=\left(\left(\varphi^{*} d V\right)^{\#}\right\rfloor\left(\varphi^{*}\left(\sum_{j=1}^{n} \bar{\partial} Q_{j} d \zeta_{j}\right)^{n-m} \wedge \varphi^{*} \Omega\left[l_{1}, \ldots, l_{m}\right]\right)\right)(0, \varphi(w)) .
\end{aligned}
$$

Symbol $*$ denotes the pullback operation.

Lemma 3.4 in [25] gives us the following estimate in $P_{|r(\zeta)|}^{i}(\zeta)$ :

$$
\begin{aligned}
& \left.\mid\left((d V)^{\#}\right\rfloor\left(\left(\sum_{j=1}^{n} \bar{\partial} Q_{j} d \zeta_{j}\right)^{n-m} \wedge \Omega\left[l_{1}, \ldots, l_{m}\right]\right)\right)(\zeta, z) \mid \\
& \leq C \sum_{\alpha, \beta} \frac{\left(2^{i}|r(\zeta)|\right)^{n-m}}{\prod_{j=1}^{n-m} \tau_{\alpha_{j}}\left(\zeta, 2^{i}|r(\zeta)|\right) \tau_{\beta_{j}}\left(\zeta, 2^{i}|r(\zeta)|\right)}\left|\operatorname{det}\left(\begin{array}{ccc}
\frac{\partial\left(l_{1} \circ \varphi\right)}{\partial \eta_{\alpha_{n-m+1}}} & \cdots & \frac{\partial\left(l_{1} \circ \varphi\right)}{\partial \eta_{\alpha_{n}}} \\
\frac{\partial\left(l_{m} \circ \varphi\right)}{\partial \eta_{\alpha_{n-m+1}}} & \cdots & \frac{\partial\left(l_{m} \circ \varphi\right)}{\partial \eta_{\alpha_{n}}}
\end{array}\right)\right| \\
& \left|\operatorname{det}\left(\begin{array}{ccc}
\frac{\partial\left(l_{1} \circ \varphi\right)}{\partial \eta_{\beta_{n-m+1}}} & \cdots & \frac{\partial\left(l_{1} \circ \varphi\right)}{\partial \eta_{\beta_{n}}} \\
\frac{\partial\left(l_{m} \circ \varphi\right)}{\partial \eta_{\beta_{n-m+1}}} & \cdots & \frac{\partial\left(l_{m} \circ \varphi\right)}{\partial \eta_{\beta_{n}}}
\end{array}\right)\right|
\end{aligned}
$$

where the sum is over all permutations $\alpha, \beta$ of $1, \ldots, n$.

This gives

$$
\int_{P_{|r(\zeta)|}^{i}(\zeta)}\left|E_{D \cap A}^{N}(\zeta, z)\right| d V(z) \leq\left(2^{i}\right)^{-N} \sum_{\alpha} \frac{V\left(P_{2^{i}|r(\zeta)|}(\zeta)\right)}{\prod_{j=1}^{n-m} \tau_{\alpha_{j}}^{2}\left(\zeta, 2^{i}|r(\zeta)|\right)}\left|\operatorname{det}\left(\begin{array}{ccc}
\frac{\partial\left(l_{1} \circ \varphi\right)}{\partial \eta_{\alpha_{n-m+1}}} & \cdots & \frac{\partial\left(l_{1} \circ \varphi\right)}{\partial \eta_{\alpha_{n}}} \\
\frac{\partial\left(l_{m} \circ \varphi\right)}{\partial \eta_{\alpha_{n-m+1}}} & \cdots & \frac{\partial\left(l_{m} \circ \varphi\right)}{\partial \eta_{\alpha_{n}}}
\end{array}\right)\right|^{2},
$$

where the sum is again over all permutations $\alpha$ of $1, \ldots, n$.

Since $V\left(P_{2^{i}|r(\zeta)|}(\zeta)\right)=\prod_{j=1}^{n} \tau_{j}^{2}\left(\zeta, 2^{i}|r(\zeta)|\right)$ and $\tau(\zeta, v, 2 \varepsilon) \lesssim$ $2 \tau(\zeta, v, \varepsilon)$, we obtain the following estimate:

$$
\begin{gathered}
\int_{P_{\mid r(\zeta)}^{i}(\zeta)}\left|E_{D \cap A}^{N}(\zeta, z)\right| d V(z) \leq C\left(2^{i}\right)^{-(N-2 m)} \\
\cdot \sum_{\alpha}\left|\operatorname{det}\left(\begin{array}{ccc}
\frac{\partial\left(l_{1} \circ \varphi\right)}{\partial \eta_{\alpha_{n-m+1}}} & \cdots & \frac{\partial\left(l_{1} \circ \varphi\right)}{\partial \eta_{\alpha_{n}}} \\
\frac{\partial\left(l_{m} \circ \varphi\right)}{\partial \eta_{\alpha_{n-m+1}}} & \cdots & \frac{\partial\left(l_{m} \circ \varphi\right)}{\partial \eta_{\alpha_{n}}}
\end{array}\right)\right|^{2}
\end{gathered}
$$

$$
\begin{aligned}
& \cdot \prod_{j=n-m+1}^{n} \tau_{\alpha_{j}}^{2}(\zeta,|r(\zeta)|) \leq C\left(2^{i}\right)^{-(N-2 m)} \mid \partial l_{1} \wedge \cdots \\
& \left.\wedge \partial l_{m}\right|_{\mathcal{N}} ^{2}(\zeta) .
\end{aligned}
$$

The last estimate follows from the fact that

$$
\begin{aligned}
\operatorname{det}\left(\begin{array}{ccc}
\frac{\partial\left(l_{1} \circ \varphi\right)}{\partial \eta_{\alpha_{n-m+1}}} & \cdots & \frac{\partial\left(l_{1} \circ \varphi\right)}{\partial \eta_{\alpha_{n}}} \\
\frac{\partial\left(l_{m} \circ \varphi\right)}{\partial \eta_{\alpha_{n-m+1}}} & \cdots & \frac{\partial\left(l_{m} \circ \varphi\right)}{\partial \eta_{\alpha_{n}}}
\end{array}\right) \\
=\left(\partial l_{1} \wedge \cdots \wedge \partial l_{m}\right)\left(v_{\alpha_{n-m+1}^{\zeta, \varepsilon}}^{\zeta,}, \ldots, v_{\alpha_{n}}^{\zeta, \varepsilon}\right) .
\end{aligned}
$$


It remains now to estimate the integral over $D \backslash P_{\varepsilon_{0}}(\zeta)$. We cannot simply say that the integral over $D \backslash P_{\varepsilon_{0}}(\zeta)$ is bounded since we claim that the integral behaves like the norm $\mid \partial l_{1} \wedge$ $\left.\cdots \wedge \partial l_{m}\right|_{\mathcal{N}} ^{2}(\zeta)$ of the form $\partial l_{1} \wedge \cdots \wedge \partial l_{m}$. Thus, in particular we claim that the integral vanishes as $\zeta$ tends to $b D$.

Naturally, if $|r(\zeta)| \gtrsim c$ for a positive constant $c$, then

$$
\int_{D}\left|E_{D \cap A}^{N}(\zeta, z)\right| d V(z) \leq C
$$

with a constant which depends only on $c$, since it can easily be proved that $|r(\zeta)+S(z, \zeta)| \gtrsim|r(\zeta)|$. Also in this case, $\mid \partial l_{1} \wedge \cdots \wedge$ $\left.\partial l_{m}\right|_{\mathcal{N}} ^{2}(\zeta) \gtrsim C$ since $\tau(\zeta, v, \varepsilon) \gtrsim \varepsilon$ uniformly for unit vectors $v$ and points $\zeta \in D$. This establishes (20) in $D \backslash P_{\varepsilon_{0}}(\zeta)$ provided $|r(\zeta)| \geq c$.

Since $|S(z, \zeta)| \gtrsim \varepsilon$ in $P_{\varepsilon}^{0}(\zeta)$, we may assume that $|r(\zeta)| \leq$ $\varepsilon_{0} / 2$ and obtain the estimate

$$
|r(\zeta)+S(z, \zeta)| \geq|S(z, \zeta)|-|r(\zeta)| \geq \varepsilon_{0} .
$$

Trivial estimates of the form $Q$ give therefore under the assumption that $|r(\zeta)| \leq \varepsilon_{0} / 2$ that

$$
\begin{aligned}
& \int_{D \backslash P_{\varepsilon_{0}}(\zeta)}\left|E_{D \cap A}^{N}(\zeta, z)\right| d V_{D \cap A} \\
& \leq C|r(\zeta)|^{N} \sum_{1 \leq j_{1}<\cdots<j_{m} \leq n}\left|\operatorname{det}\left(\begin{array}{ccc}
\frac{\partial l_{1}}{\partial \zeta_{j_{1}}} & \cdots & \frac{\partial l_{1}}{\partial \zeta_{j_{m}}} \\
& \cdots & \\
\frac{\partial l_{m}}{\partial \zeta_{j_{1}}} & \cdots & \frac{\partial l_{m}}{\partial \zeta_{j_{m}}}
\end{array}\right)\right|^{2} .
\end{aligned}
$$

Since $|r(\zeta)| \lesssim \tau(\zeta, v,|r(\zeta)|)$ uniformly for unit vectors $v$, we also have

$$
\begin{gathered}
\int_{D \backslash P_{\varepsilon_{0}}(\zeta)}\left|E_{D \cap A}^{N}(\zeta, z)\right| d V_{D \cap A}(z) \\
\leq C\left|\partial l_{1} \wedge \cdots \wedge \partial l_{m}\right|_{\mathcal{N}}^{2}(\zeta),
\end{gathered}
$$

provided $N \geq 2 m$. This completes the proof.

We can now prove Theorem 2.

Proof of Theorem 2. It follows from Fubini's Theorem and Lemma 6 that if $N$ is sufficiently large, then

$$
E_{D \cap A}^{N}: H^{1}(D \cap A, \omega) \longrightarrow H^{1}(D),
$$

where

$$
\omega=\left|\partial l_{1} \wedge \cdots \wedge l_{m}\right|_{\mathcal{N}}^{2} d V_{D \cap A}
$$

Since $\left|\partial l_{1} \wedge \cdots \wedge l_{m}\right|_{\mathcal{N}}(\zeta) \leq C(-r(\zeta))^{m / M}$, it follows immediately that $H^{1}(D \cap A) \subset H^{1}(D \cap A, \omega)$. It remains to notice that $H^{1}(D \cap A) \neq H^{1}(D \cap A, \omega)$. In other words, in order to complete the proof it suffices to show that there exists a holomorphic function satisfying

$$
\int_{D}|f|(-r)^{m / M} d V<\infty
$$

which does not belong to $H^{1}(D \cap A)$. We consider the convex domain $D \cap A$ in $A \cong \mathbb{C}^{n-m}$. We may assume that $\mathfrak{R} z_{1}=1$ is a plane tangent to $b(D \cap A)$ at $(1,0, \ldots, 0)$ and $D \cap A$ lies on the left-hand side of $\Re z_{1}=1$. Since $D \cap A$ is convex, it suffices to take $\left(1-z_{1}\right)^{\alpha}$ for suitable $\alpha<0$.

We intend to complete the proof of Theorem 1. For this, we need the following lemma.

Lemma 7. Assume that $D$ is a bounded convex domain of finite type in $\mathbb{C}^{n}, n>1$ defined by a function $r$ which is smooth in $\mathbb{C}^{n} \backslash\{0\}$, convex in $\mathbb{C}^{n}$ and such that $d r \neq 0$ on $b D$. For the variety $A=A\left(l_{1}, \ldots, l_{m}\right)$, consider the operator $E_{D \cap A}^{N}$. Assume that

$$
1+\frac{1}{p-1}>\min \{n-m, m\}>1 .
$$

For any $\epsilon>0$ such that

$$
c a 1+(1-\epsilon) \frac{1}{p-1} \geq \min \{n-m, m\}
$$

there exists a constant $C_{\epsilon}$ such that

$$
\begin{aligned}
& \int_{D \cap A}\left|E_{D \cap A}^{N}(\zeta, z)\right|\left|\partial l_{1} \wedge \cdots \wedge \partial l_{m}\right|_{\mathcal{N}}^{(1-\epsilon)(2 /(p-1))} \\
& \cdot(\zeta) d V_{D \cap A}(\zeta) \leq C_{\epsilon}
\end{aligned}
$$

In the proof of Lemma 7, we will use the following two lemmas.

Lemma 8. There exists an open set $U \supset b D$ and a constant $c$ such that if $z, \zeta \in U$ and $\zeta \in P_{c}(z) \backslash P_{2^{i}|r(z)|}(z)$ with $2^{i}|r(z)|<c$, then

$$
|r(\zeta)+S(z, \zeta)| \gtrsim 2^{i}|r(z)|
$$

Lemma 9. Let $\Omega$ be a $(m, 0)$-covector at $\zeta \in D$. There exists a constant $C$ such that if $\zeta \in P_{2^{i}|r(z)|}(z), z \in D, i \in \mathbb{N}_{0}$, then

$$
\begin{aligned}
|\Omega|_{\mathcal{N}}(\zeta) \leq & C \sum_{1 \leq j_{1}<\cdots<j_{m} \leq n}\left|\Omega\left(v_{j_{1}}^{z, 2^{i}|r(z)|}, \ldots, v_{j_{m}}^{z, 2^{i}|r(z)|}\right)\right| \\
& \cdot \prod_{k=1}^{m} \tau_{k}\left(z, 2^{i}|r(z)|\right),
\end{aligned}
$$

where $\left(v_{1}^{z, 2^{i}|r(z)|}, \ldots, v_{n}^{z, 2^{i}|r(z)|}\right)$ is the $2^{i}|r(z)|$-extremal basis at $z$.

We omit the proof of Lemma 8 since it can be proved in the same way as Lemma 4.2 in [25] or Lemma 3.3 in [32]. We concentrate on the proof of Lemma 9.

Proof of Lemma 9. Let $u_{1}, \ldots, u_{m}$ be unit vectors. We can write

$$
u_{k}=\sum_{j=1}^{n} \alpha_{k j} v_{j}^{z, 2^{i}|r(z)|}, \quad k=1, \ldots, m
$$


and also

$$
\begin{aligned}
& \Omega\left(u_{1}, \ldots, u_{m}\right) \\
& \quad=\sum_{j_{1}, \ldots, j_{m}=1}^{n} \alpha_{1 j_{1}} \cdots \alpha_{m j_{m}} \Omega\left(v_{j_{1}}^{z, 2^{i}|r(z)|}, \ldots, v_{j_{m}}^{z, 2^{i}|r(z)|}\right) .
\end{aligned}
$$

If $\zeta \in P_{2^{i}|r(z)|}(z)$, then $|r(\zeta)| \lesssim 2^{i}|r(z)|$ and, as a result, uniformly for any unit vector $v$

$$
\tau(\zeta, v,|r(\zeta)|) \lesssim \tau\left(\zeta, v, 2^{i}|r(z)|\right)
$$

when $\zeta \in P_{2^{i}|r(z)|}(z)$. If $\zeta \in P_{\varepsilon}(z)$, then uniformly for any unit vector $v$

$$
\tau(\zeta, v, \varepsilon) \sim \tau(z, v, \varepsilon)
$$

This is Proposition 2.3 in [18].

Thus, if $\zeta \in P_{2^{i}|r(z)|}(z)$, then for any unit vector $v$

$$
\tau(\zeta, v,|r(\zeta)|) \leqslant \tau\left(z, v, 2^{i}|r(z)|\right) .
$$

It is proved in [18] (Proposition 2.2, cf. also [30]) that if $u=$ $\sum_{j=1}^{n} \alpha_{j} v_{j}^{z, \varepsilon}$, where $v_{1}^{z, \varepsilon}, \ldots, v_{n}^{z, \varepsilon}$ is the $\varepsilon$-extremal basis at $z$, then

$$
\frac{1}{\tau(z, u, \varepsilon)} \sim \sum_{j=1}^{n} \frac{\left|\alpha_{j}\right|}{\tau_{j}(z, \varepsilon)} .
$$

Thus, we have $\tau(z, u, \varepsilon) \lesssim \tau_{j}(z, \varepsilon) /\left|\alpha_{j}\right|$ if $\alpha_{j} \neq 0$. For any nonzero vectors $u_{1}, \ldots, u_{m}$, we can therefore estimate

$$
\begin{aligned}
& \left|\Omega\left(u_{1}, \ldots, u_{m}\right)\right| \prod_{k=1}^{m} \tau\left(\zeta, u_{k},|r(\zeta)|\right) \\
& \quad \leq C \sum_{1 \leq j_{1}<\cdots<j_{m} \leq n} \frac{\left|\alpha_{1 j_{1}} \cdots \alpha_{m j_{m}}\right|}{\left|\alpha_{1 j_{1}} \cdots \alpha_{m j_{m}}\right|} \mid \Omega\left(v_{j_{1}}^{z, 2^{i}|r(z)|}, \ldots,\right. \\
& \left.v_{j_{m}}^{z, 2^{i}|r(z)|}\right) \mid \prod_{k=1}^{m} \tau_{j_{k}}\left(z, 2^{i}|r(z)|\right),
\end{aligned}
$$

which completes the proof.

Proof of Lemma 7. Naturally,

$$
\begin{aligned}
& \int_{(D \cap A) \backslash P_{\varepsilon_{0}}(z)}\left|E_{D \cap A}^{N}(\zeta, z)\right|\left|\partial l_{1} \wedge \cdots \wedge \partial l_{m}\right|_{\mathcal{N}}^{(1-\epsilon)(2 /(p-1))} \\
& \cdot(\zeta) d V_{D \cap A}(\zeta)<C
\end{aligned}
$$

with an independent constant $C$. This follows from the fact that $|S(z, \zeta)| \gtrsim \varepsilon$ in $P_{\varepsilon}^{0}(z)$ (Lemma $\left.3.2[22]\right)$ and that

$$
|r(\zeta)+S(z, \zeta)| \gtrsim|r(\zeta)|
$$

which holds true for every $z, \zeta \in D$. Hence, we need to show that under our assumptions

$$
\begin{aligned}
& \int_{D \cap A \cap P_{\varepsilon_{0}}(z)}\left|E_{D \cap A}^{N}(\zeta, z)\right|\left|\partial l_{1} \wedge \cdots \wedge \partial l_{m}\right|_{\mathcal{N}}^{(1-\epsilon)(2 /(p-1))} \\
& \cdot(\zeta) d V_{D \cap A}(\zeta)
\end{aligned}
$$

is uniformly bounded for $z \in D$. This time we therefore fix $z \in D$ and consider the cover $P_{|r(z)|}(z), P_{|r(z)|}^{i}(z), i=$ $0, \ldots, C\left\lceil\log _{2}\left(\varepsilon_{0} /|r(z)|\right)\right\rceil$ of the polydisc $P_{\varepsilon_{0}}(z)$. Again we work with a typical term of $E_{D \cap A}^{N}(\cdot, \cdot)$ keeping the same notation. It follows from Lemma 8 that if $\zeta \in P_{|r(z)|}^{i}(z)$, then

$$
\begin{aligned}
& \left.\left|E_{D \cap A}^{N}(\zeta, z)\right| \leq C\left(2^{i}|r(z)|\right)^{-(n-m)} \mid(d V)^{\#}\right\rfloor \\
& \quad \cdot\left(\left(\sum_{j=1}^{n} \bar{\partial} Q_{j}(z, \zeta) \wedge d \zeta_{j}\right)^{n-m} \wedge \Omega\left[l_{1}, \ldots, l_{m}\right]\right) .
\end{aligned}
$$

Let $\left(v_{1}, \ldots, v_{n}\right)=\left(v_{1}^{z, 2^{i}|r(z)|}, \ldots, v_{n}^{2^{i}|r(z)|}\right)$ be the $2^{i}|r(z)|-$ extremal basis at $z$. Let $\left(\eta_{1}, \ldots, \eta_{n}\right)$ be the corresponding coordinates of a point $\zeta$ and let $\Phi$ be a unitary map such that $\zeta=\Phi(\eta)+z:=\varphi(\eta)$; that is, $\eta=\Phi^{\star}(\zeta-z)$. Using the map, $\varphi$ we change coordinates in the integral

$$
\begin{aligned}
& \left.\int_{D \cap A \cap P_{|r(z)|}^{i}(z)} \mid(d V)^{\#}\right\rfloor \\
& \cdot\left(\left(\sum_{j=1}^{n} \bar{\partial} Q_{j}(z, \zeta) \wedge d \zeta_{j}\right)^{n-m} \wedge \Omega\left[l_{1}, \ldots, l_{m}\right]\right) \mid \\
& \cdot\left|\partial l_{1} \wedge \cdots \wedge \partial l_{m}\right|_{\mathcal{N}}^{(1-\epsilon)(2 /(p-1))}(\zeta) d V_{D \cap A}(\zeta) .
\end{aligned}
$$

Functoriality of the contraction and Lemma3.3 in [22] together with (54) yield the following estimate:

$$
\begin{aligned}
& \int_{D \cap A \cap P_{|r(z)|}^{i}(z)}\left|E_{D \cap A}(\zeta, z)\right|\left|\partial l_{1} \wedge \cdots \wedge \partial l_{m}\right|_{\mathcal{N}}^{(1-\varepsilon)(2 /(p-1))} \\
& \cdot(\zeta) d V_{D \cap A}(\zeta) \\
& \leq C \sum_{\alpha} \frac{1}{\prod_{j=1}^{n-m} \tau_{\alpha_{j}}^{2}\left(z, 2^{i}|r(z)|\right)} \\
& \quad \cdot \int_{\varphi^{-1}(D \cap A) \cap\left\{\left|\eta_{j}\right|<\tau_{j}\left(z, 2^{i}|r(z)|\right)\right\}}\left|D_{\alpha_{n-m+1}, \ldots, \alpha_{n}}\right|^{2} \\
& \cdot\left|\partial l_{1} \wedge \cdots \wedge \partial l_{m}\right|_{\mathcal{N}}^{(1-\varepsilon)(2 /(p-1))}(\varphi(\eta)) \varphi^{*}\left(d V_{D \cap A}\right)
\end{aligned}
$$

$\cdot(\eta)$

where $\varphi^{*} d V_{D \cap A}$ denotes the pullback of the volume form $d V_{D \cap A}$ and

$$
D_{\alpha_{n-m+1}, \ldots, \alpha_{n}}=\operatorname{det}\left(\begin{array}{ccc}
\frac{\partial\left(l_{1} \circ \varphi\right)}{\partial \eta_{\alpha_{n-m+1}}} & \cdots & \frac{\partial\left(l_{1} \circ \varphi\right)}{\partial \eta_{\alpha_{n}}} \\
\frac{\partial\left(l_{m} \circ \varphi\right)}{\partial \eta_{\alpha_{n-m+1}}} & \cdots & \frac{\partial\left(l_{m} \circ \varphi\right)}{\partial \eta_{\alpha_{n}}}
\end{array}\right)
$$


The sum is over all permutations $\alpha$ of the set $1, \ldots, n$. We use Lemma 9 and obtain the following estimate:

$$
\begin{aligned}
& \left|\partial l_{1} \wedge \cdots \wedge \partial l_{m}\right|_{\mathcal{N}}^{(1-\epsilon)(2 /(p-1))}(\zeta) \\
& \quad \leq C\left(\sum_{1 \leq j_{1}<\cdots<j_{m} \leq n}\left|D_{j_{1}, \ldots, j_{m}}\right|\right. \\
& \left.\quad \cdot \prod_{k=1}^{m} \tau_{j_{k}}\left(z, 2^{i}|r(z)|\right)\right)^{(1-\epsilon)(2 /(p-1))} \\
& \quad \leq C \sum_{1 \leq j_{1}<\cdots<j_{m} \leq n}\left|D_{j_{1}, \ldots, j_{m}}\right| \\
& \quad \cdot \prod_{k=1}^{m} \tau_{j_{k}}^{(1-\epsilon)(2 /(p-1))}\left(z, 2^{i}|r(z)|\right),
\end{aligned}
$$

since $\zeta \in P_{2^{i}|r(z)|}(z)$. The last estimate is a consequence of the fact that $t \mapsto t^{\alpha}$ is a convex function provided $\alpha \geq 1$ and by the assumption $(1-\epsilon)(2 /(p-1)) \geq 2$. It is important to realize that estimate (58) is made with respect to the same $2^{i}|r(z)|$ extremal basis at $z$ as for instance in (56).

We have therefore the following estimate of the integral:

$$
\begin{aligned}
& \int_{D \cap A \cap P_{|r(z)|}^{i}(z)}\left|E_{D \cap A}(\zeta, z)\right|\left|\partial l_{1} \wedge \cdots \wedge \partial l_{m}\right|_{\mathcal{N}}^{(1-\epsilon)(2 /(p-1))} \\
& \cdot(\zeta) d V_{D \cap A}(\zeta) \\
& \quad \leq C \sum_{\alpha} \sum_{1 \leq j_{1}<\cdots<j_{m} \leq n} \frac{\prod_{k=1}^{m} \tau_{j_{k}}^{(1-\epsilon)(2 /(p-1))}\left(z, 2^{i}|r(z)|\right)}{\prod_{j=1}^{n-m} \tau_{\alpha_{j}}^{2}\left(z, 2^{i}|r(z)|\right)} \\
& \cdot\left|D_{\alpha_{n-m+1}, \ldots, \alpha_{n}}\right|^{2}\left|D_{j_{1}, \ldots, j_{m}}\right|^{(1-\epsilon)(2 /(p-1))} \\
& \quad \cdot \int_{\varphi^{-1}(D \cap A) \cap\left\{\left|\eta_{j}\right|<\tau_{j}\left(z, 2^{i}|r(z)|\right)\right\}} \varphi^{*}\left(d V_{D \cap A}\right) .
\end{aligned}
$$

Since $|x|^{2}|y|^{2 a} \leq|x|^{2+2 a}+|y|^{2+2 a}$, we also have

$$
\begin{aligned}
& \int_{D \cap A \cap P_{|r(z)|}^{i}(z)}\left|E_{D \cap A}(\zeta, z)\right|\left|\partial l_{1} \wedge \cdots \wedge \partial l_{m}\right|_{\mathcal{N}}^{(1-\epsilon)(2 /(p-1))} \\
& \cdot(\zeta) d V_{D \cap A}(\zeta) \\
& \quad \leq C \sum_{\alpha} \sum_{1 \leq j_{1}<\cdots<j_{m} \leq n} \frac{\prod_{k=1}^{m} \tau_{j_{k}}^{(1-\epsilon)(2 /(p-1))}\left(z, 2^{i}|r(z)|\right)}{\prod_{j=1}^{n-m} \tau_{\alpha_{j}}^{2}\left(z, 2^{i}|r(z)|\right)} \\
& \cdot\left(\left|D_{\alpha_{n-m+1}, \ldots, \alpha_{n}}\right|^{2+(1-\epsilon)(2 /(p-1))}\right. \\
& \left.\quad+\left|D_{j_{1}, \ldots, j_{m}}\right|^{2+(1-\epsilon)(2 /(p-1))}\right) \\
& \quad \cdot \int_{\varphi^{-1}(D \cap A) \cap\left\{\left|\eta_{j}\right|<\tau_{j}\left(z, 2^{i}|r(z)|\right)\right\}} \varphi^{*}\left(d V_{D \cap A}\right) .
\end{aligned}
$$

Therefore, we are led to estimate the following expression:

$$
\begin{aligned}
& \left|D_{\alpha_{1}, \ldots, \alpha_{m}}\right|^{2+(1-\epsilon)(2 /(p-1))} \\
& \quad \cdot \int_{\varphi^{-1}(D \cap A) \cap\left\{\left|\eta_{j}\right|<\tau_{j}\left(z, 2^{i}|r(z)|\right)\right\}} \varphi^{*}\left(d V_{D \cap A}\right) .
\end{aligned}
$$

In other words, we need to estimate $\varphi^{*}\left(d V_{D \cap A}\right)$ on $\varphi^{-1}(D \cap A)$. Here, we use Wirtinger's formula according to which

$$
d V_{D \cap A}=\left.\left(\frac{\sqrt{-1}}{2(n-m) !}\right)^{n-m}\left(\sum_{j=1}^{n} d \zeta_{j} \wedge d \bar{\zeta}_{j}\right)^{n-m}\right|_{D \cap A}
$$

As a result,

$$
\begin{aligned}
\varphi^{*} & \left(d V_{D \cap A}\right) \\
& =\left.\left(\frac{\sqrt{-1}}{2(n-m) !}\right)^{n-m}\left(\sum_{j=1}^{n} d \eta_{j} \wedge d \bar{\eta}_{j}\right)^{n-m}\right|_{\varphi^{-1}(D \cap A)} .
\end{aligned}
$$

In order to have control over (61), we need to get rid of variables $\eta_{\alpha_{1}}, \ldots, \eta_{\alpha_{m}}$ in the form $\varphi^{*}\left(d V_{D \cap A}\right)$. Since $\partial\left(l_{j} \circ \varphi\right)=$ $0, j=1, \ldots, m$, on $\varphi^{-1}(D \cap A)$, we have on $\varphi^{-1}(D \cap A)$ the following equality:

$$
\begin{gathered}
\left(\begin{array}{ccc}
\frac{\partial\left(l_{1} \circ \varphi\right)}{\partial \eta_{\alpha_{1}}} & \cdots & \frac{\partial\left(l_{1} \circ \varphi\right)}{\partial \eta_{\alpha_{m}}} \\
\frac{\partial\left(l_{m} \circ \varphi\right)}{\partial \eta_{\alpha_{1}}} & \cdots & \frac{\partial\left(l_{m} \circ \varphi\right)}{\partial \eta_{\alpha_{m}}}
\end{array}\right)\left(\begin{array}{c}
d \eta_{\alpha_{1}} \\
\ldots \\
d \eta_{\alpha_{m}}
\end{array}\right) \\
=-\left(\begin{array}{c}
\frac{\partial\left(l_{1} \circ \varphi\right)}{\partial \eta_{\alpha_{m+1}}} d \eta_{\alpha_{m+1}}+\cdots+\frac{\partial\left(l_{1} \circ \varphi\right)}{\partial \eta_{\alpha_{n}}} d \eta_{\alpha_{n}} \\
\cdots \\
\frac{\partial\left(l_{m} \circ \varphi\right)}{\partial \eta_{\alpha_{m+1}}} d \eta_{\alpha_{m+1}}+\cdots+\frac{\partial\left(l_{m} \circ \varphi\right)}{\partial \eta_{\alpha_{n}}} d \eta_{\alpha_{n}}
\end{array}\right) .
\end{gathered}
$$

Thus, whenever $D_{\alpha_{1}, \ldots, \alpha_{m}} \neq 0$, we obtain the estimate

$$
\begin{aligned}
& \varphi^{*}\left(d V_{D \cap A}\right) \\
& \leq C \frac{d \Re \eta_{\alpha_{m+1}} \wedge d \Im \eta_{\alpha_{m+1}} \wedge \cdots \wedge d \Re \eta_{\alpha_{n}} \wedge d \Im \eta_{\alpha_{n}}}{\left|D_{\alpha_{1}, \ldots, \alpha_{m}}\right|^{2 \min \{m, n-m\}}} .
\end{aligned}
$$

Under the assumption that $2+(1-\epsilon)(2 /(p-1)) \geq 2 \min \{m, n-$ $m\}$, this gives

$$
\begin{aligned}
& \left|D_{\alpha_{1}, \ldots, \alpha_{m}}\right|^{2+(1-\epsilon)(2 /(p-1))} \\
& \cdot \int_{\varphi^{-1}(D \cap A) \cap\left\{\left|\eta_{j}\right|<\tau_{j}\left(z, 2^{i}|r(z)|\right)\right\}} \varphi^{*}\left(d V_{D \cap A}\right) \\
& \quad \leq C \prod_{l=m+1}^{n} \tau_{\alpha_{l}}^{2}\left(z, 2^{i}|r(z)|\right),
\end{aligned}
$$

where $\alpha$ is a permutation of the set $1, \ldots, n$. 
From (60), we therefore obtain

$$
\begin{aligned}
& \int_{D \cap A \cap P_{|r(z)|}^{i}(z)}\left|E_{D \cap A}(\zeta, z)\right|\left|\partial l_{1} \wedge \cdots \wedge \partial l_{m}\right|_{\mathcal{N}}^{(1-\epsilon)(2 /(p-1))} \\
& \cdot(\zeta) d V_{D \cap A}(\zeta) \\
& \quad \leq C \sum_{\alpha} \sum_{1 \leq j_{1}<\cdots<j_{m} \leq n} \frac{\prod_{k=1}^{m} \tau_{j_{k}}^{(1-\epsilon)(2 /(p-1))}\left(z, 2^{i}|r(z)|\right)}{\prod_{j=1}^{n-m} \tau_{\alpha_{j}}^{2}\left(z, 2^{i}|r(z)|\right)} \\
& \quad\left(\prod_{j=1}^{n-m} \tau_{\alpha_{j}}^{2}\left(z, 2^{i}|r(z)|\right)+\prod_{k=m+1}^{n} \tau_{j_{k}}^{2}\left(z, 2^{i}|r(z)|\right)\right),
\end{aligned}
$$

where $\left\{j_{m+1}, \ldots, j_{n}\right\} \cup\left\{j_{1}, \ldots, j_{m}\right\}=\{1, \ldots, n\}$. Thus,

$$
\begin{aligned}
& \int_{D \cap A \cap P_{|r(z)|}^{i}(z)}\left|E_{D \cap A}(\zeta, z)\right|\left|\partial l_{1} \wedge \cdots \wedge \partial l_{m}\right|_{\mathcal{N}}^{(1-\epsilon)(2 /(p-1))} \\
& \cdot(\zeta) d V_{D \cap A}(\eta) \\
& \leq C \sum_{\alpha} \sum_{1 \leq j_{1}<\cdots<j_{m} \leq n}\left(\prod_{k=1}^{m} \tau_{j_{k}}^{(1-\epsilon)(2 /(p-1))}\left(z, 2^{i}|r(z)|\right)\right. \\
& +\frac{\prod_{k=1}^{n} \tau_{k}^{2}\left(z, 2^{i}|r(z)|\right)}{\prod_{j=1}^{n-m} \tau_{\alpha_{j}}^{2}\left(z, 2^{i}|r(z)|\right)} \\
& \left.\cdot \prod_{k=1}^{m} \tau_{j_{k}}^{(1-\epsilon)(2 /(p-1))-2}\left(z, 2^{i}|r(z)|\right)\right) .
\end{aligned}
$$

Since $\tau(\zeta, v,|r(\zeta)|) \lesssim|r(\zeta)|^{1 / M}$, we obtain for some $A>0$ that

$$
\begin{aligned}
& \int_{D \cap A \cap P_{|r(z)|}^{i}(z)}\left|E_{D \cap A}(\zeta, z)\right|\left|\partial l_{1} \wedge \cdots \wedge \partial l_{m}\right|_{\mathcal{N}}^{(1-\epsilon)(2 /(p-1))} \\
& \cdot(\zeta) d V_{D \cap A}(\zeta) \leq C\left(2^{i}|r(z)|\right)^{A}
\end{aligned}
$$

Thus,

$$
\begin{aligned}
& \int_{D \cap A \cap P_{\varepsilon_{0}}(z)}\left|E_{D \cap A}(\zeta, z)\right|\left|\partial l_{1} \wedge \cdots \wedge \partial l_{m}\right|_{\mathcal{N}}^{(1-\epsilon)(2 /(p-1))} \\
& \cdot(\zeta) d V_{D \cap A}(\zeta) \\
& \quad \leq C \sum_{i=0}^{C\left[\log _{2}\left(\varepsilon_{0} /|r(z)|\right)\right]}\left(2^{i}|r(z)|\right)^{A} \leq C
\end{aligned}
$$

This completes the proof.

We can now prove Theorem 1.

Proof of Theorem 1. If $1+1 /(p-1)>\min \{m, n-m\}$, then there exists $\epsilon>0$ such that $1+(1-\epsilon)(1 /(p-1)) \geq \min \{m, n-m\}$. Choose such a small positive number $\epsilon$.

As has already been stated, we apply Proposition 4 . Thus, we set $d \nu=d V, d \mu=d V_{D \cap A}, h_{1}(\zeta):=\left|\partial l_{1} \wedge \cdots \wedge \partial l_{m}\right|_{\mathcal{N}}^{2 / p}(\zeta)$, $h_{2} \equiv 1$, and $W(\zeta):=\left|\partial l_{1} \wedge \cdots \wedge \partial l_{m}\right|_{\mathcal{N}}^{2 \epsilon}(\zeta)$. Then, it follows from Lemmas 6 and 7 and Proposition 4 that

$$
E_{D \cap A}^{N}: H^{p}(D \cap A, d \omega) \longrightarrow H^{p}(D),
$$

where $d \omega=\left|\partial l_{1} \wedge \cdots \wedge \partial l_{m}\right|_{\mathcal{N}}^{2 \epsilon} d V_{D \cap A}$, provided $N$ is sufficiently large.

Since $\left|\partial l_{1} \wedge \cdots \wedge \partial l_{m}\right|_{\mathcal{N}} \lesssim(-r)^{m / M}$, we have that $d \omega \lesssim$ $(-r)^{2 \epsilon(m / M)} d V_{D \cap A}$. Since $D$ is convex, it is therefore easy to show that $H^{p}(D \cap A) \varsubsetneqq H^{p}(D \cap A, d \omega)$.

Lastly we provide sketch of Theorem 3.

Proof of Theorem 3. We again apply Schur criteria, that is, Proposition 4. This time however with $W \equiv 1$. In view of Lemma 6 , we set $h_{1}(\zeta):=\left|\partial l_{1} \wedge \cdots \wedge \partial l_{m}\right|^{2 / p}(\zeta), h_{2} \equiv 1$.

In order to complete the proof, we need to know that

$$
\begin{gathered}
\int_{D \cap A}\left|E_{D \cap A}^{N}(\zeta, z)\right|\left|\partial l_{1} \wedge \cdots \wedge \partial l_{m}\right|^{2 /(p-1)} \\
\cdot(\zeta) d V_{D \cap A}(\zeta) \leq C .
\end{gathered}
$$

For the estimates in the proof of Lemma 7 to work, we must have $p \leq 2$. Indeed, only under this assumption we can have control over the expression

$$
\frac{\prod_{k=1}^{m} \tau_{j_{k}}^{2 /(p-1)}\left(z, 2^{i}|r(z)|\right)}{\prod_{j=1}^{n-m} \tau_{\alpha_{j}}^{2}\left(z, 2^{i}|r(z)|\right)}
$$

which appears in (60). We also need to have $2+(2 /(p-1)) \geq$ $2 \min \{m, n-m\}$ to obtain estimate (66). The readers easily convince themselves that if $p \leq 2$ and $\min \{m, n-m\} \leq 2$, then the estimates in the proof of Lemma 7 can be repeated.

\section{Conflict of Interests}

The author declares that there is no conflict of interests regarding the publication of this paper.

\section{References}

[1] T. Ohsawa and K. Takegoshi, "On the extension of $L^{2}$ holomorphic functions," Mathematische Zeitschrift, vol. 195, pp. 197-204, 1987.

[2] K. Diederich and E. Mazzilli, "Extension and restriction of holomorphic functions," Annales de l'Institut Fourier, vol. 47, no. 4, pp. 1079-1099, 1997.

[3] J.-P. Demailly, C. D. Hacon, and M. Paun, "Extension theorems, non-vanishing and the existence of good minimal models," Acta Mathematica, vol. 210, no. 2, pp. 203-259, 2013.

[4] G. M. Henkin, "Continuation of bounded holomorphic functions from submanifolds in general position in a strictly pseudoconvex domain," Izvestiya Akademii Nauk SSSR. Seriya Matematicheskaya, vol. 36, pp. 540-567, 1972.

[5] E. Amar, "Extension de fonctions holomorphes et courants," Bulletin des Sciences Mathématiques, vol. 107, no. 1, pp. 25-48, 1983.

[6] K. Diederich and E. Mazzilli, "Extension of bounded holomorphic functions in convex domains," Manuscripta Mathematica, vol. 105, no. 1, pp. 1-12, 2001.

[7] W. Alexandre, "Problémes d'extension dans les domaines convexes de type fini," Mathematische Zeitschrift, vol. 253, no. 2, pp. 263-280, 2006. 
[8] A. Cumenge, "Extension dans des classes de Hardy de fonctions holomorphes et estimations de type 'measures de Carleson' pour l'equation $\bar{\partial}$,' Annales de l'institut Fourier, vol. 33, pp. 5997, 1983.

[9] J. J. Kohn, "Boundary behaviour of $\bar{\partial}$ on weakly pseudoconvex manifolds of dimension two," Journal of Differential Geometry, vol. 6, pp. 523-542, 1972.

[10] J. J. Kohn, "Subellipticity of the $\bar{\partial}$-Neumann problem on pseudo-convex domains: sufficient conditions," Acta Mathematica, vol. 142, pp. 79-122, 1979.

[11] D. Catlin, "Necessary conditions for subellipticity of the $\bar{\partial}$ Neumann problem," Annals of Mathematics, vol. 117, pp. 141-171, 1983.

[12] D. Catlin, "Subelliptic estimates for the $\bar{\partial}$-Neumann problem on pseudoconvex domains," Annals of Mathematics, vol. 126, pp. 131-191, 1987.

[13] J. P. D'Angelo, "Real hypersurfaces, orders of contact, and applications," Annals of Mathematics, vol. 115, no. 3, pp. 615-637, 1982.

[14] H. P. Boas and E. J. Straube, "On equality of line and variety type of real hypersurfaces in $\mathbb{C}^{n}$," The Journal of Geometric Analysis, vol. 2, no. 2, pp. 95-98, 1992.

[15] J. D. McNeal, "Convex domains of finite type," Journal of Functional Analysis, vol. 108, no. 2, pp. 361-373, 1992.

[16] J. Y. Yu, "Multitypes of convex domains," Indiana University Mathematics Journal, vol. 41, no. 3, pp. 837-849, 1992.

[17] J. Bruna, A. Nagel, and S. Wainger, "Convex hypersurfaces and Fourier transforms," Annals of Mathematics, vol. 127, no. 2, pp. 333-365, 1988.

[18] J. D. McNeal, "Estimates on the Bergman kernels of convex domains," Advances in Mathematics, vol. 109, no. 1, pp. 108-139, 1994.

[19] J. D. McNeal and E. M. Stein, "The Szegö projection on convex domains," Mathematische Zeitschrift, vol. 224, no. 4, pp. 519-553, 1997.

[20] J. Bruna, P. Charpentier, and Y. Dupain, "Zero varieties for the Nevanlinna class in convex domains of finite type in $\mathbb{C}^{\mathrm{n}}$," Annals of Mathematics, vol. 147, no. 2, pp. 391-415, 1998.

[21] K. Diederich and J. E. Fornæss, "Support functions for convex domains of finite type," Mathematische Zeitschrift, vol. 230, no. 1, pp. 145-164, 1999.

[22] B. Fischer, " $L^{p}$ estimates on convex domains of finite type," Mathematische Zeitschrift, vol. 236, no. 2, pp. 401-418, 2001.

[23] T. Hefer, "Hölder and $L^{p}$ estimates for $\bar{\partial}$ on convex domains of finite type depending on Catlin's multitype," Mathematische Zeitschrift, vol. 242, pp. 367-398, 2002.

[24] T. Hefer, "Extremal bases and Hölder estimates for $\bar{\partial}$ on convex domains of finite type," The Michigan Mathematical Journal, vol. 52, no. 3, pp. 573-602, 2004.

[25] K. Diederich, B. Fischer, and J. E. Fornæss, "Hölder estimates on convex domains of finite type," Mathematische Zeitschrift, vol. 232, no. 1, pp. 43-61, 1999.

[26] B. Fischer, "Nonisotropic Hölder estimates on convex domains of finite type," The Michigan Mathematical Journal, vol. 52, no. 1, pp. 219-239, 2004.

[27] B. Berndtsson and M. Andersson, "Henkin-Ramirez formulas with weight factors," Annales de l'Institut Fourier, vol. 32, pp. 91110, 1982.

[28] B. Berndtsson, "A formula for interpolation and division in $\mathbb{C}^{n}$," Mathematische Annalen, vol. 263, no. 4, pp. 399-418, 1983.
[29] S. G. Krantz and S.-Y. Li, "Duality theorems for Hardy and Bergman spaces on convex domains of finite type in $\mathbb{C}^{n}$," Annales de l'institut Fourier, vol. 45, pp. 1305-1327, 1995.

[30] N. Nikolov, P. Pflug, and P. J. Thomas, "On different extremal bases for $\mathbb{C}$-convex domains," Proceedings of the American Mathematical Society, vol. 141, no. 9, pp. 3223-3230, 2013.

[31] K. H. Zhu, Operator Theory in Function Spaces, vol. 139 of Monographs and Textbooks in Pure and Applied Mathematics, Marcel Dekker, New York, NY, USA, 1990.

[32] K. Diederich and E. Mazzilli, "Zero varieties for the Nevanlinna class on all convex domains of finite type," Nagoya Mathematical Journal, vol. 163, pp. 215-227, 2001. 


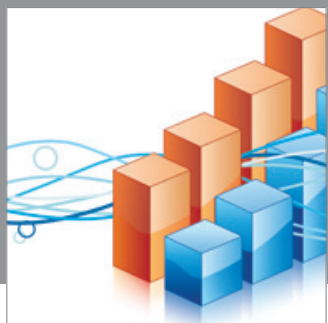

Advances in

Operations Research

mansans

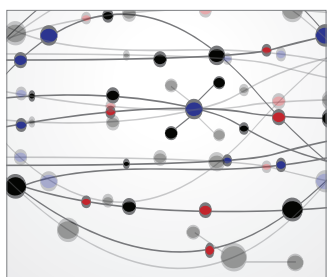

The Scientific World Journal
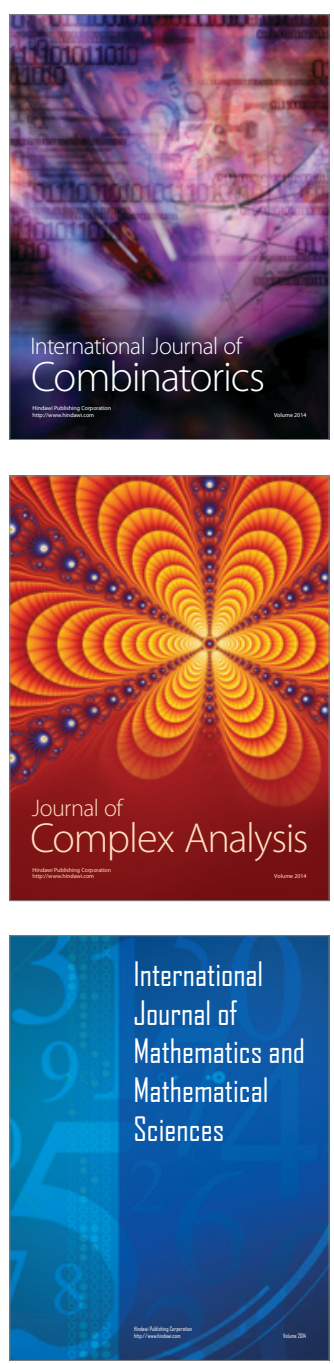
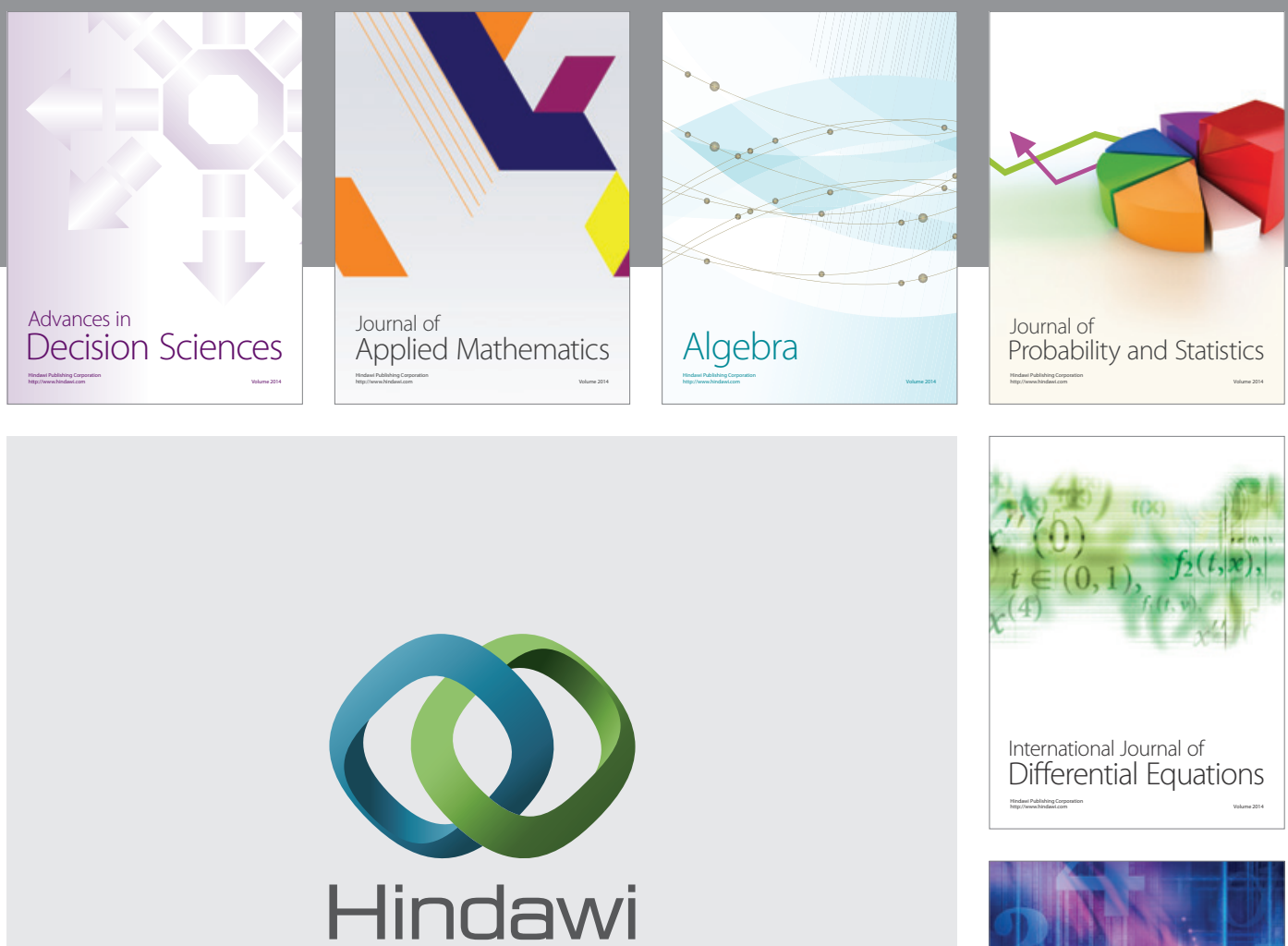

Submit your manuscripts at http://www.hindawi.com
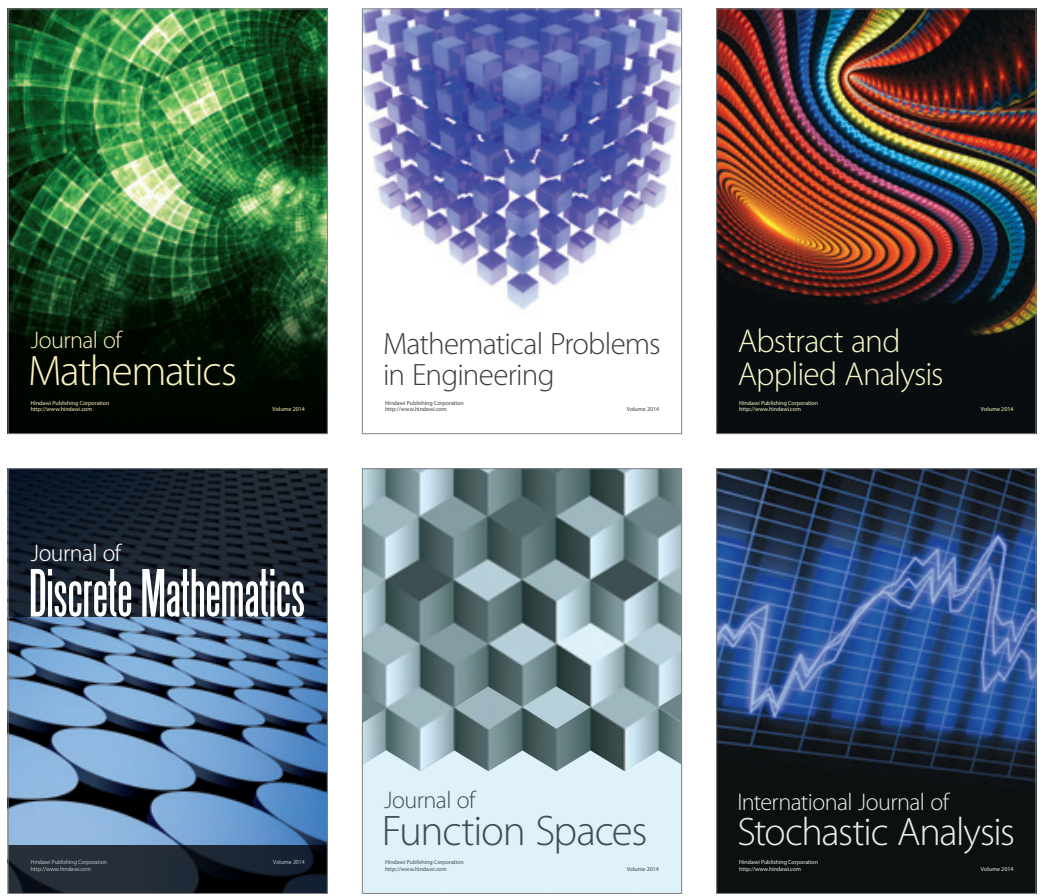

Journal of

Function Spaces

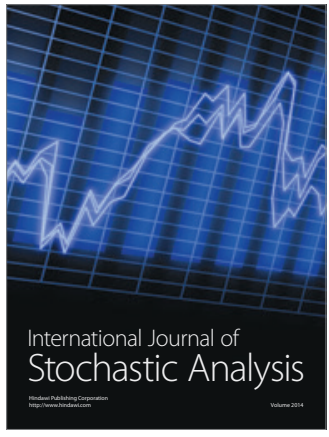

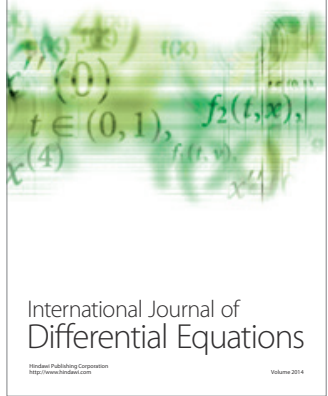
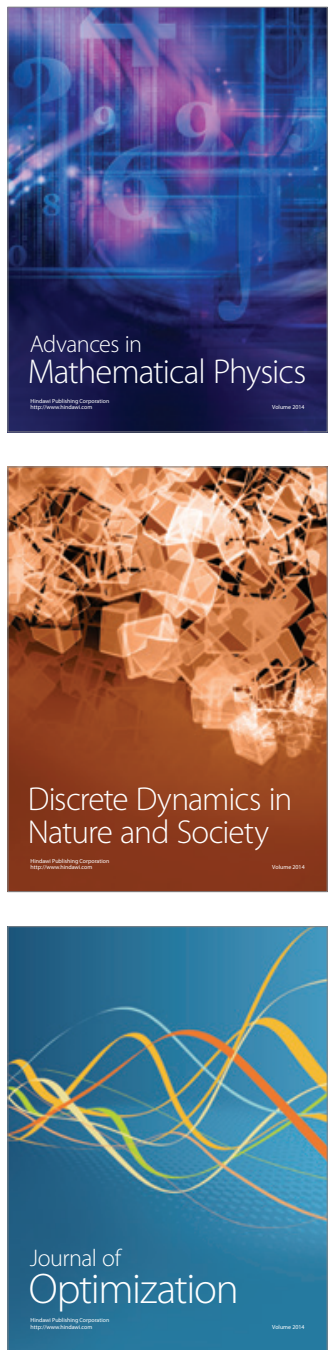